\title{
PAPER \\ A Comparative Study on Bandwidth and Noise for Pre-Emphasis and Post-Equalization in Visible Light Communication
}

\author{
Dong YAN ${ }^{\dagger}$, Member, Xurui MAO ${ }^{\dagger \dagger a)}$, Sheng $\mathrm{XIE}^{\dagger \dagger \dagger}$, Jia CONG ${ }^{\dagger}$, Dongqun $\mathrm{HAN}^{\dagger}$, \\ and Yicheng $\mathrm{WU}^{\dagger}$, Nonmembers
}

\begin{abstract}
SUMMARY This paper presents an analysis of the relationship between noise and bandwidth in visible light communication (VLC) systems. In the past few years, pre-emphasis and post-equalization techniques were proposed to extend the bandwidth of VLC systems. However, these bandwidth extension techniques also influence noise and sensitivity of the VLC systems. In this paper, first, we build a system model of VLC transceivers and circuit models of pre-emphasis and postequalization. Next, we theoretically compare the bandwidth and noise of three different transceiver structures comprising a single pre-emphasis circuit, a single post-equalization circuit and a combination of pre-emphasis and post-equalization circuits. Finally, we validate the presented theoretical analysis using experimental results. The result shows that for the same resonant frequency, and for high signal-to-noise ratio (S/N), VLC systems employing post-equalization or pre-emphasis have the same bandwidth extension ability. Therefore, a transceiver employing both the pre-emphasis and post-equalization techniques has a bandwidth $\sqrt{2}$ times the bandwidth of the systems employing only the pre-emphasis or post-equalization. Based on the theoretical analysis of noise, the VLC system with only active preemphasis shows the lowest noise, which is a good choice for low-noise systems. The result of this paper may provide a new perspective of noise and sensitivity of the bandwidth extension techniques in VLC systems.

key words: visible light communication (VLC), pre-emphasis, postequalization, noise, bandwidth
\end{abstract}

\section{Introduction}

VLC technology using light emitting diodes (LED) transmits information via LED lighting equipment, such as traffic lights, car lights, street lights and LED screens. These devices can emit high-speed flashing light and dark signal that cannot be perceived by human eyes [1], [2]. Compared with the wireless local area network (WLAN), which has dominant position in the field of wireless communications, VLC is a novel optical wireless communication (OWC) based on LED technology. VLC technology is expected to release the RF spectrum and reduce the WLAN overloading in some applications [3]. It can achieve transmission data rates of up to hundreds of megabytes per second without using a radio frequency base station. Using computers and mo-

\footnotetext{
Manuscript received September 30, 2019.

Manuscript revised January 19, 2020.

Manuscript publicized February 25, 2020.

†The authors are with School of Electrical and Information Engineering, Tianjin University, Tianjin 300072, China.

${ }^{\dagger}$ The author is with State Key Laboratory on Integrated Optoelectronics, Institute of Semiconductors, Chinese Academy of Sciences, Beijing 100083, China.

${ }^{\dagger \dagger}$ The author is with School of Microelectronics, Tianjin University, Tianjin 300072, China.

a) E-mail: maoxurui@semi.ac.cn

DOI: 10.1587/transcom.2019EBP3206
}

bile terminals with integrated transceiver module, we can download and upload data including high-definition images and movies at any time as long as suitable light sources are available. Herald Haas and his research team at the University of Edinburgh proposed the concept of "light fidelity (Li-Fi)", defined as "the subset of VLC that exhibits highspeed, bidirectional, and fully networked communication". Li-Fi is considered as a "bright spot" for VLC applications, and it is expected to complement wireless fidelity (Wi-Fi) [4]-[6].

Due to a low cut-off frequency of the phosphor-based LED, implementing high data rate transmission is a challenge. The time constant of LED is so large that their bandwidth is typically in the range of few $\mathrm{MHz}$ [7]. The bandwidth of the white-light LED is below $3 \mathrm{MHz}$, while the bandwidth of the blue light LED is roughly $14 \mathrm{MHz}$. To enhance the bandwidth of the VLC systems, several solutions have been proposed in the literature. The authors in [8] proposed inserting a blue-filter before the photo-detector to improve the photocurrent of the receiver by eliminating other components in the light. Remaining-carriers sweep-out circuits [9], infrared uplink [10], gallium nitride $(\mathrm{GaN})$ based $\mu$ LEDs [11] and micro-LEDs [12], [13] have been proposed to solve the challenge in indoor VLC system design. The main approach to improve bandwidth and data rate of a VLC system is by using the pre-emphasis technique [7], [14]-[21] and post-equalization technique [22], [23]. Implementing a VLC system to meet the higher data rate requirements and to accommodate more application scenarios typically employs pre-emphasis and post-equalization circuits at the same time [24], [25]. High-speed data transmission can be realized by VLC systems based on RGB LEDs [26], [27] Spectrallyefficient modulation-techniques were also proposed to increase the data rate, such as carrier-less amplitude and phase (CAP) modulation [28], [29], orthogonal frequency division multiplexing (OFDM) modulation [30], discrete multi-tone (DMT) modulation [31].

Most of the studies up to now aimed at improving the bandwidth and data rate of VLC systems and enhancing the BER of indoor VLC systems. Except for improving the data-rate of VLC systems, the research of [7], [22]-[24] also include the frequency response analysis and 3-dB point calculations of pre-emphasis circuit and post-equalization circuits. However, a theoretical analysis showing the relationship between noise and bandwidth of a VLC system is still unavailable. This paper provides a theoretical analysis of the 
relationship between noise and bandwidth in VLC systems in this paper.

In Sect. 2, we provide the system modeling and bandwidth analysis of three VLC systems employing different equalization schemes. In Sect. 3, we derive the $\mathrm{S} / \mathrm{N}$ expressions for three different VLC systems employing only preemphasis, post-equalization, or both pre-emphasis and postequalization, respectively. We theoretically analyse the performance of the three systems in Sect. 4, and provide experimental results validating our theoretical analysis, in Sect. 5.

\section{Transceiver System Model and Bandwidth Analysis}

In order to characterize noise and bandwidth, we established transceiver system models of VLC using the three equalization techniques.

As shown in Fig. 1, the system models have been built using the essential blocks containing LED, free-space channel, photo-detector, trans-impedance preamplifier (TIA), post-equalizer (PEQ) and pre-emphasis (PEM). As shown in Fig. 1(a), the first VLC system employs only PEQ and the second VLC system shown in Fig. 1(b) employs only PEM. The third VLC system considered in this paper is shown in Fig. 1(c), and employs both PEM and PEQ.

In order to highlight the key elements and simplify the theoretical analysis, the signal path of LED, free-space channel, detector and TIA is considered as a whole or a black box. $N_{P}$ represents the equivalent input noise of the signal path. $H_{P}(\omega)$ represents the transfer function of the signal path. Similarly, $N_{P E M}$ and $N_{P E Q}$ represent the equivalent input noise of PEM and PEQ, respectively. $H_{P E M}(\omega)$ and $H_{P E Q}(\omega)$ represent the transfer functions of PEM and PEQ, respectively.

Next, the analysis of the bandwidth performance is presented as follow.

In practical situation, the cut-off frequency of the detector and TIA are much greater than the 3-dB bandwidth of LED and the compensation frequency of equalizers. Hence, the detector and TIA can be regarded as all-pass filter. Therefore, the transfer function of $H_{P}(\omega)$ is mainly decided by LED and free-space channel.

The limited bandwidth of LED and free-space chan-
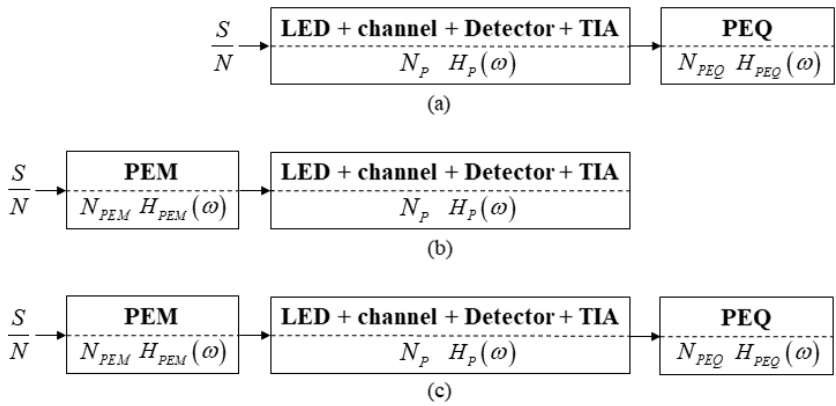

Fig. 1 Block diagram of three transceiver systems with different equalization schemes. (a) Post-equalization system, (b) pre-emphasis system, and (c) pre-emphasis + post-equalization system. nel can equivalently be modeled using a low-pass filter. An equivalent low-pass circuit is shown in Fig. 2(a), and the corresponding frequency response can be expressed as

$$
H_{P}(\omega)=\frac{\frac{1}{j \omega C}}{R+\frac{1}{j \omega C}}=\frac{1}{1+j \omega R C}=\frac{1}{1+j \frac{\omega}{\omega_{P}}}
$$

Where $\omega_{P}$ presents the cut-off frequency of the signal path. The cut-off frequency is given by

$$
\omega_{P}=\frac{1}{R C}
$$

The pre-emphasis circuit and post-equalization circuit can be considered as high-pass filters. Their equivalent circuit is shown in Fig. 2(b). The transfer function of the highpass filter can be expressed as

$$
H_{H P}(\omega)=\frac{j \omega L}{R+j \omega L}=\frac{1}{1+\frac{R}{j \omega L}}=\frac{1}{1+\frac{\omega_{H P}}{j \omega}}
$$

Where $\omega_{H P}$ presents the cut-off frequency of the equalizers. The cut-off frequency is given by

$$
\omega_{H P}=\frac{R}{L}
$$

First, we consider the compensation bandwidth of a VLC system with a single post-equalizer, whose equivalent circuit model is depicted in Fig. 3. The principle of the equalization is such that the equivalent circuit of the postequalizer and the signal-path resonate at the compensation frequency, which is equal to the $3-\mathrm{dB}$ bandwidth of the circuit.

Because the value of $R$ is usually much smaller than

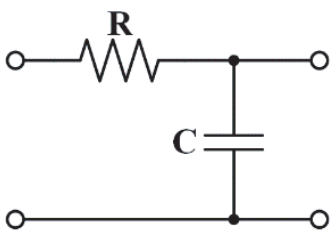

(a) (b)

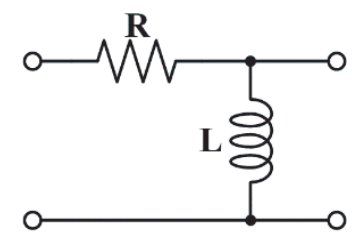

Fig. 2 Equivalent circuits of (a) signal path of LED, free-space channel, detector and TIA; (b) pre-emphasis and post-equalization.

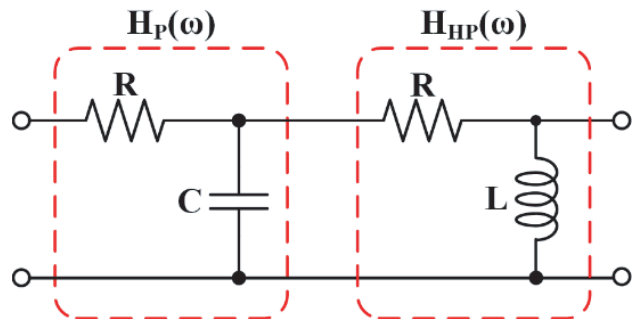

Fig. 3 Equivalent circuit for the compensation of single equalizer. 


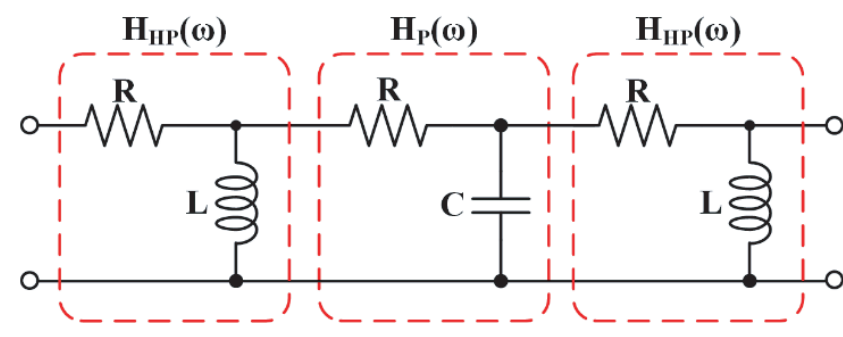

Fig. 4 The equivalent circuit model of using both pre-emphasis and postequalization compensation.

the values of $C$ and $L$, the resonant frequency of the postequalizer can be expressed as

$$
\omega_{1}=\frac{1}{\sqrt{L C}}
$$

Next, we consider the compensation bandwidth of a VLC system with a single pre-emphasis. For this case also, the equivalent circuit of the VLC system is similar to the one shown in Fig. 3. Hence, the compensation principle is the same as for the VLC system with a single post-equalizer, and its resonant frequency is given by

$$
\omega_{2}=\frac{1}{\sqrt{L C}}
$$

Finally, for the VLC system with both pre-emphasis and post-equalizer, the equivalent circuit model is shown in Fig. 4. The maximum frequency of the equalization compensation for the circuit in Fig. 4 is $\omega_{3}$. Since the value of $R$ is usually much smaller than the values of $C$ and $L, \omega_{3}$ can be derived as

$$
\omega_{3}=\sqrt{\frac{2}{L C}}
$$

As we can observe, the resonant frequency of the system, using both the pre-emphasis and post-equalization circuits is $\sqrt{2}$ times higher than that of the systems with a single pre-emphasis or post-equalization circuit. The conclusion is based on the same value of $\mathrm{L}$ for three different systems.

However, the value of $\mathrm{L}$ of the pre-emphasis circuit and the post-equalization circuit does not need to be the same. If the value of $\mathrm{L}$ of the pre-emphasis circuit can decrease to one half of L, only the pre-emphasis circuit is sufficient to improve the bandwidth and the post-equalization circuit is unnecessary. Otherwise, if the value of $\mathrm{L}$ of the postemphasis circuit can decrease to one half of $\mathrm{L}$, only the postemphasis circuit is sufficient to improve the bandwidth and the pre-equalization circuit is unnecessary. The value of $\mathrm{L}$ of the pre-emphasis, post-equalization, and both pre-emphasis and post-equalization circuits can be selected independently.

The optimal value of $\mathrm{L}$ is related to the frequency response of signal-path. In the practical situations, the frequency response of signal-path is always non-linear. It's difficult to get the optimal value of $\mathrm{L}$ by theoretical analysis. The optimal value of $\mathrm{L}$ can be achieved by software simulation and experimental tuning.

\section{S/N Analysis}

The power spectral density of the output signal through a linear network is the product of the input signal power spectral density and the square of the transfer function [32], which can be expressed as

$$
\mathbf{S}_{o}=S_{i} \prod_{i=1}^{n}\left|H_{i}(\omega)\right|^{2} .
$$

Similarly, the power spectral density of the noise through a linear network is the sum of the product of the input signal power spectral density and the square of the transfer function [33], which can be expressed as

$$
\mathbf{S}_{n}=\sum_{i=1}^{n} \prod_{j=i}^{n} S_{i}\left|H_{j}(\omega)\right|^{2}
$$

The noise analysis of communication systems is customarily based on white noise, the power spectral density of which is independent of the operating frequency [32]. Since the analysis in this paper is the transfer characteristics and accumulation of noise, the conclusion is also available for shot noise and thermal noise.

\subsection{Post-Equalization Receiver (System A)}

For the post-equalization receiver shown in the Fig. 1(a), the power of signal and noise entering the system are denoted by $S$ and $N$, respectively. When entering the equalizer, both signal and noise components get equalized.

The power spectral density of the output signal and noise are expressed as $S_{A}$ and $N_{A}{ }^{\prime}$ :

$$
\begin{aligned}
& S_{A}=S\left|H_{P}(\omega)\right|^{2}\left|H_{P E Q}(\omega)\right|^{2} \\
& N_{A}{ }^{\prime}=\left[\left(N+N_{P}\right)\left|H_{P}(\omega)\right|^{2}+N_{P E Q}\right]\left|H_{P E Q}(\omega)\right|^{2}
\end{aligned}
$$

The SNR after the equalization is given by

$$
S N R_{A}=\frac{S\left|H_{P}(\omega)\right|^{2}\left|H_{P E Q}(\omega)\right|^{2}}{\left[\left(N+N_{P}\right)\left|H_{P}(\omega)\right|^{2}+N_{P E Q}\right]\left|H_{P E Q}(\omega)\right|^{2}}
$$

In order to compare the noise characteristics of three different systems, we normalized the numerator of SNR expression.

$$
S N R_{A}=\frac{S}{N+N_{P}+\frac{1}{\left|H_{P}(\omega)\right|^{2}} \cdot N_{P E Q}}
$$

Therefore, the normalized output noise is:

$$
N_{A}=N+N_{P}+\frac{1}{\left|H_{P}(\omega)\right|^{2}} \cdot N_{P E Q}
$$

Comparison of three systems is presented in Sect. 4. 


\subsection{Pre-Emphasis Receiver (System B)}

For the pre-emphasis receiver shown in the Fig. 1(b), signal and noise components are both equalized in the first stage by the pre-emphasis circuit. The equivalent input noise of the pre-emphasis is denoted by $N_{P E M}$, as the same as postequalization receiver. The power spectral density of the output signal is given by

$$
S_{B}=S\left|H_{P E M}(\omega)\right|^{2}\left|H_{P}(\omega)\right|^{2}
$$

The power spectral density of output noise can be expressed as

$$
N_{B}{ }^{\prime}=\left[\left(N+N_{P E M}\right)\left|H_{P E M}(\omega)\right|^{2}+N_{P}\right]\left|H_{P}(\omega)\right|^{2}
$$

The SNR of system B can be expressed as

$$
S N R_{B}=\frac{S\left|H_{P E M}(\omega)\right|^{2}\left|H_{P}(\omega)\right|^{2}}{\left[\left(N+N_{P E M}\right)\left|H_{P E M}(\omega)\right|^{2}+N_{P}\right]\left|H_{P}(\omega)\right|^{2}}
$$

In order to compare the noise characteristics of three different systems, we normalized the numerator of SNR expression.

$$
S N R_{B}=\frac{S}{N+N_{P E M}+\frac{1}{\left|H_{P E M}(\omega)\right|^{2}} \cdot N_{P}}
$$

Therefore, the normalized output noise is:

$$
N_{B}=N+N_{P E M}+\frac{1}{\left|H_{P E M}(\omega)\right|^{2}} \cdot N_{P}
$$

Comparison of three systems is presented in Sect. 4.

3.3 Pre-Emphasis and Post-Equalization Receiver (System C)

The VLC system with both pre-emphasis and postequalization is the same as pre-emphasis receiver except that a post-equalizer added to the system. The power spectral density of the output signal can be expressed as

$$
S_{C}=S\left|H_{P E M}(\omega)\right|^{2}\left|H_{P}(\omega)\right|^{2}\left|H_{P E Q}(\omega)\right|^{2}
$$

The power spectral density of output noise is given by

$$
N_{C^{\prime}}=\left(N_{B}+N_{P E Q}\right)\left|H_{P E Q}(\omega)\right|^{2}
$$

The SNR of system C can be expressed as

$$
S N R_{C}=\frac{S\left|H_{P E M}(\omega)\right|^{2}\left|H_{P}(\omega)\right|^{2}\left|H_{P E Q}(\omega)\right|^{2}}{\left(N_{B}+N_{P E Q}\right)\left|H_{P E Q}(\omega)\right|^{2}}
$$

In order to compare the noise characteristics of three different systems, we normalized the numerator of SNR expression.

$$
S N R_{C}=\frac{S}{N+N_{P E M}+\frac{1}{\left|H_{P E M}(\omega)\right|^{2}} \cdot N_{P}+\frac{1}{\left|H_{P E M}(\omega)\right|^{2}\left|H_{P}(\omega)\right|^{2}} \cdot N_{P E Q}}
$$

Therefore, the normalized output noise is:

$N_{C}=N+N_{P E M}+\frac{1}{\left|H_{P E M}(\omega)\right|^{2}} \cdot N_{P}+\frac{1}{\left|H_{P E M}(\omega)\right|^{2}\left|H_{P}(\omega)\right|^{2}} \cdot N_{P E Q}$

Comparison of three systems is presented in Sect. 4.

\section{Analysis of Noise and Bandwidth of Pre-Equilibrium and Post-Equilibrium}

The VLC system with only pre-emphasis or postequalization has the same bandwidth as shown in Eq. (4) and Eq. (5). Furthermore, the bandwidth of the VLC system employing both pre-emphasis and post-equalization is $\sqrt{2}$ times bandwidth of the single equalization systems.

For a clearer and more intuitive comparison, we listed the coefficient of noise elements in Table 1.

The content of this section is arranged as follows: at first, we compare $N_{B}$ and $N_{C}$; then, we compare $N_{A}$ and $N_{B}$; then, we compare $N_{A}$ and $N_{C}$; at last, the comparison results are summarized into a table.

4.1 The Comparison of Only PEM System $\left(N_{B}\right)$ and PEM+PEQ System $\left(N_{C}\right)$

According to Eq. (19) and Eq. (24), $N_{C}$ include the noise component of $\frac{1}{\left|H_{P E M}(\omega)\right|^{2}\left|H_{P}(\omega)\right|^{2}} \cdot N_{P E Q}$, which does not exist in $N_{B}$. Obviously, $N_{>} C N_{B}$.

4.2 The Comparison of Only PEQ System $\left(N_{A}\right)$ and Only PEM System $\left(N_{B}\right)$

Except for the same noise component of $N$, the critical problem is to compare $N_{P}+\frac{1}{\left|H_{P}(\omega)\right|^{2}} \cdot N_{P E Q}$ and $\frac{1}{\left|H_{P E M}(\omega)\right|^{2}} \cdot N_{P}+N_{P E M}$. The comparison result is decided by $N_{P}, N_{P E M}, N_{P E Q}, H_{P}(\omega)$ and $H_{P E M}(\omega)$.

If the signal path performance is bad, which means $N_{P}$ is much larger than $N_{P E M}$ and $N_{P E Q}$. The comparison is focused on $N_{P}$ and $\frac{1}{\left|H_{P E M}(\omega)\right|^{2}} \cdot N_{P}$. For the passive

Table 1 Comparison of normalized noise.

\begin{tabular}{ccccc}
\hline Weight of & $N$ & $N_{P E M}$ & $N_{P}$ & $N_{P E Q}$ \\
\hline PEQ & 1 & 0 & 1 & $\frac{1}{\left|H_{P}(\omega)\right|^{2}}$ \\
PEM & 1 & 1 & $\frac{1}{\left|H_{P E M}(\omega)\right|^{2}}$ & 0 \\
PEQ+PEM & 1 & 1 & $\frac{1}{\left|H_{P E M}(\omega)\right|^{2}}$ & $\frac{1}{\left|H_{P E M}(\omega)\right|^{2}\left|H_{P}(\omega)\right|^{2}}$ \\
\hline
\end{tabular}


PEM, the transfer function $\left|H_{P E M}(\omega)\right|^{2}<1$, which leads to $N_{P}<\frac{1}{\left|H_{P E M}(\omega)\right|^{2}} \cdot N_{P}$, the comparison result is $N_{A}<N_{B}$. For the active PEM, the transfer function $\left|H_{P E M}(\omega)\right|^{2}>1$, which leads to $N_{P}>\frac{1}{\left|H_{P E M}(\omega)\right|^{2}} \cdot N_{P}$, the comparison result is $N_{A}>N_{B}$.

If the signal path performance is good, which means $N_{P}, N_{P E M}, N_{P E Q}$ are on the same order of magnitude. The comparison is focused on $H_{P}(\omega)$ and $H_{P E M}(\omega)$. In practical situation, the transfer function of signal path $\left|H_{P}(\omega)\right|^{2}<1$. For the passive PEM, both of $H_{P}(\omega)$ and $H_{P E M}(\omega)$ are less than 1, which leads to $N_{A} \approx N_{B}$. For the active PEM, the transfer function $\left|H_{P E M}(\omega)\right|^{2}>1$, which leads to $N_{A}>N_{B}$.

\subsection{The Comparison of Only PEQ System $\left(N_{A}\right)$ and PEM+PEQ System $\left(N_{C}\right)$}

Except for the same noise component of $N$, the problem is comparing $N_{P}+\frac{1}{\left|H_{P}(\omega)\right|^{2}} \cdot N_{P E Q}$ and $\frac{1}{\left|H_{P E M}(\omega)\right|^{2}} \cdot N_{P}+N_{P E M}+$ $\frac{1}{\left|H_{P E M}(\omega)\right|^{2}\left|H_{P}(\omega)\right|^{2}} \cdot N_{P E Q}$. The comparison result is decided by $N_{P}, N_{P E M}, N_{P E Q}, H_{P}(\omega)$ and $H_{P E M}(\omega)$.

If the signal path performance is bad, which means $N_{P}$ is much larger than $N_{P E M}$ and $N_{P E Q}$. The comparison is focused on $N_{P}$ and $\frac{1}{\left|H_{P E M}(\omega)\right|^{2}} \cdot N_{P}$. For the passive PEM, the transfer function $\left|H_{P E M}(\omega)\right|^{2}<1$, which leads to $N_{P}<\frac{1}{\left|H_{P E M}(\omega)\right|^{2}} \cdot N_{P}$, the comparison result is $N_{A}<N_{C}$. For the active PEM, the transfer function $\left|H_{P E M}(\omega)\right|^{2}>1$, which leads to $N_{P}>\frac{1}{\left|H_{P E M}(\omega)\right|^{2}} \cdot N_{P}$, the comparison result is $N_{A}>N_{C}$.

If the signal path performance is good, which means $N_{P}, N_{P E M}, N_{P E Q}$ are on the same order of magnitude. The comparison is focused on $H_{P}(\omega)$ and $H_{P E M}(\omega)$. In practical situation, the transfer function of signal path $\left|H_{P}(\omega)\right|^{2}<1$. For the passive PEM, both of $H_{P}(\omega)$ and $H_{P E M}(\omega)$ are less than 1 , which leads to $N_{A}<N_{C}$. For the active PEM, the transfer function $\left|H_{P E M}(\omega)\right|^{2}>1$, which leads to $N_{A}>N_{C}$.

\subsection{Comparison Results}

Based on the analysis above, we can draw the conclusion in Table 2.

In most of the practical situations, the noise performance of signal path is bad. The VLC system with only active pre-emphasis (system B) is the first choice. If the first plan is unavailable, we prefer the VLC system with only post-equalization (system A) than the VLC system of only passive pre-emphasis.

If the noise performance of signal path is good, the VLC system with only active pre-emphasis (system B) still

Table 2 Summarized comparison results.

\begin{tabular}{ccc}
\hline & Bad signal path & Good signal path \\
\hline Passive PEM & $N_{A}<N_{B}<N_{C}$ & $N_{A} \approx N_{B}<N_{C}$ \\
Active PEM & $N_{B}<N_{C}<N_{A}$ & $N_{B}<N_{C}<N_{A}$ \\
\hline
\end{tabular}

is the safe choice. If this plan is unavailable, both the VLC system with only post-equalization (system $\mathrm{A}$ ) and the VLC system of only passive pre-emphasis (system B) is OK.

The pre-emphasis compensates frequency before the LED, and hence the noise of $N_{P}$ is not affected significantly. This results in a smaller value of total noise at the output. By contrast, the post-equalization compensates frequency after the TIA, resulting in amplifying the noise of $N_{P}$. However, the influence of the equalizer noise is reduced.

From the view of noise analysis, the transfer function of post-equalization $H_{P E Q}(\omega)$ does not affect the output noise. Because post-equalization is the last stage of the system. However, the transfer function of post-equalization has an effect on the 3-dB bandwidth of the system.

The noise of the system using both pre-emphasis and post-equalization (system $\mathrm{C}$ ) is significantly higher. However, the bandwidth of the system employing both preemphasis and post-equalization is larger than the other two systems. Moreover, compensation bandwidth of the system is $\sqrt{2}$ times larger than single equalization systems.

When we decide the structure of VLC systems, we should consider the bandwidth and noise at the same time. Only wide bandwidth is not sufficient for high-speed data transmission. The VLC system of wide bandwidth (like system C), however, the noise performance is poor, which results in low data-rate. The VLC system with relatively narrow bandwidth (like system B), however, the noise performance is good, which results in high data-rate.

The analysis described here provides a theoretical basis for designing the pre-emphasis and the post-equalization optical receivers.

\section{Experimental Results}

According to the previously described VLC system, we set up the experimental system to test the performance of different methods. For this purpose, we use the pre-emphasis and post-equalization circuits shown in Fig. 5.

In Fig. 5(a), $\mathrm{R}_{1} \sim \mathrm{R}_{5}, \mathrm{C}_{2}$ and $\mathrm{Q}_{1}$ (NXP BFU520) constitute the pre-emphasis circuit. The transistor $\mathrm{Q}_{2}(\mathrm{NXP}$ BFU520) and resistances $R_{6} \sim R_{8}$ compose the amplifier and amplify the equalized signal. The capacitors $\mathrm{C}_{1}, \mathrm{C}_{3}$ and $\mathrm{C}_{4}$ act as the DC-block capacitors with large capacitance $(10 \mu \mathrm{F})$. The values of components in the pre-emphasis circuit are given as follow: $R_{1}=90 \mathrm{k} \Omega, R_{2}=10 \mathrm{k} \Omega, R_{3}=$ $100 \Omega, \mathrm{R}_{4}=90 \Omega, \mathrm{R}_{5}=2 \Omega, \mathrm{C}_{2}=150 \mathrm{pF}, \mathrm{R}_{6}=90 \mathrm{k} \Omega$,
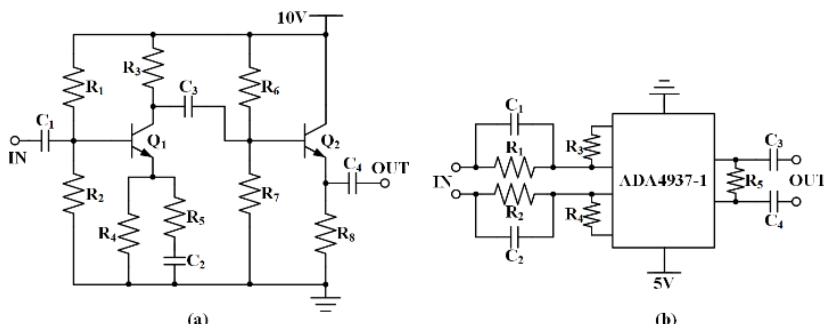

(b)

Fig. 5 Circuits of (a) pre-emphasis and (b) post-equalization. 


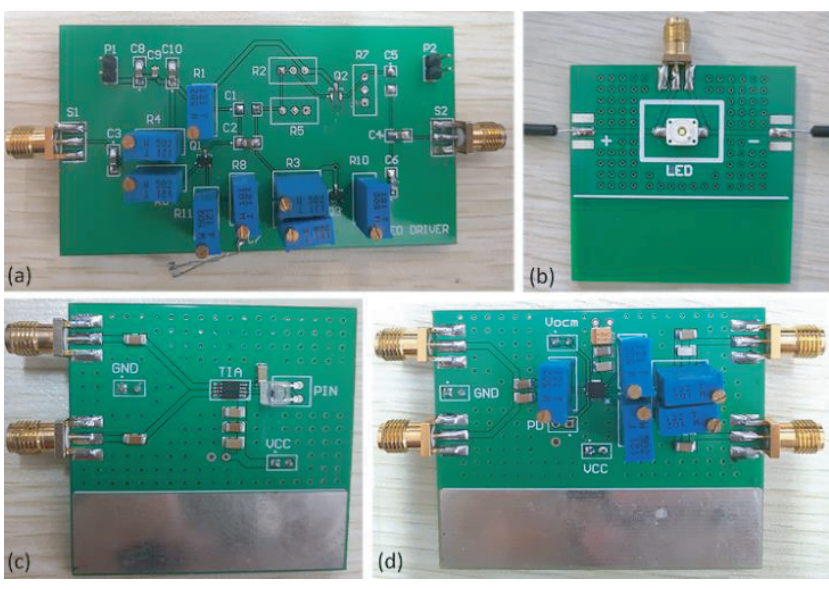

Fig. 6 The PCB used in the experiment (a) the board with pre-emphasis (b) the LED board (c) the photodetector board with TIA (d) the board with post-equalization.

$\mathrm{R}_{7}=10 \mathrm{k} \Omega, \mathrm{R}_{8}=72 \Omega$. The current consumption of this circuit is $5 \mathrm{~mA}$.

In Fig. 5(b), the post-equalizer consists of a differential amplifier (ADA4937-1) and two R-C equalization networks. $\mathrm{R}_{1}, \mathrm{C}_{1}, \mathrm{R}_{2}$ and $\mathrm{C}_{2}$ act as the R-C equalization networks. $\mathrm{R}_{3}$ and $\mathrm{R}_{4}$ are the feedback resistors of the differential amplifier. The capacitors $C_{3}$ and $C_{4}$ act as the DC-block capacitors with large capacitance $(10 \mu \mathrm{F}) . \mathrm{R}_{5}$ is the load resistor of amplifier. The values of components in the pre-emphasis circuit are given as follow: $R_{1}=R_{2}=100 \Omega, C_{1}=C_{2}=$ $85 \mathrm{pF}, \mathrm{R}_{3}=\mathrm{R}_{4}=200 \Omega, \mathrm{R}_{5}=1 \mathrm{k} \Omega$.

The overall system illustrated in Fig. 6 consists of the transmitter and the receiver circuits. The transmitter circuit includes the pre-emphasis amplifier circuit and LED shown in Fig. 6(a) and (b), respectively. The pre-emphasis circuit is implemented according to Fig. 5(a), while the board in Fig. 6(b) contains only a single LED (OSRAM LCW W5AM). The receiver circuit comprises the PIN photoelectric detector (Hamamatsu S10784) with a trans-impedance amplifier (MAX3665CUB) and a post-equalizer. The detector with TIA is shown in Fig. 6(c) and the post-equalizer corresponding to the circuit in Fig. 5(b) is illustrated in Fig. 6(d). The experimental system contains only download link without return path.

The main instruments used for testing include optical fixed platform, DC power supply and network analyzer (Agilent E5062A). The VLC experiment link is depicted in Fig. 7. The experiment is done indoor. We used the Thorlabs PM100D optical power and energy meter to measure the ambient light power. The measured optical power of ambient light is $80.2 \mu \mathrm{W}$ at $450 \mathrm{~nm}, 69.3 \mu \mathrm{W}$ at $520 \mathrm{~nm}, 57.4 \mu \mathrm{W}$ at $630 \mathrm{~nm}$. The transmission distance is $1.5 \mathrm{~m}$. The network analyzer, which can scan from $300 \mathrm{kHz}$ to $3 \mathrm{GHz}$, sends a sine wave to the transmitter. The input power of signal is $0 \mathrm{dBm}$ with $50 \Omega$ output resistance. According to the definition of S-parameter, $S_{21}=P_{\text {OUT }} / P_{I N}$. The $\mathrm{S} 21$ curve is the power gain as a function of frequency, which presents the frequency response performance.

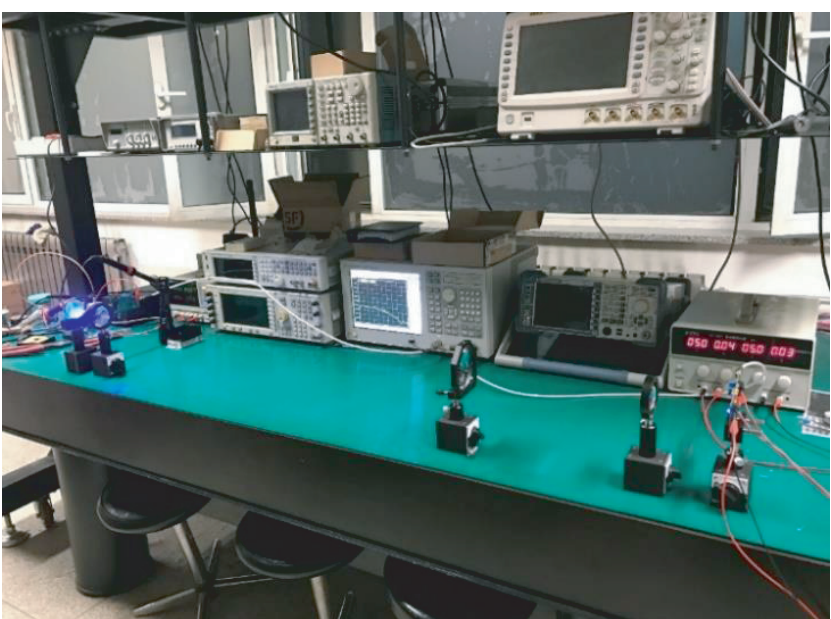

Fig. 7 VLC experiment link.
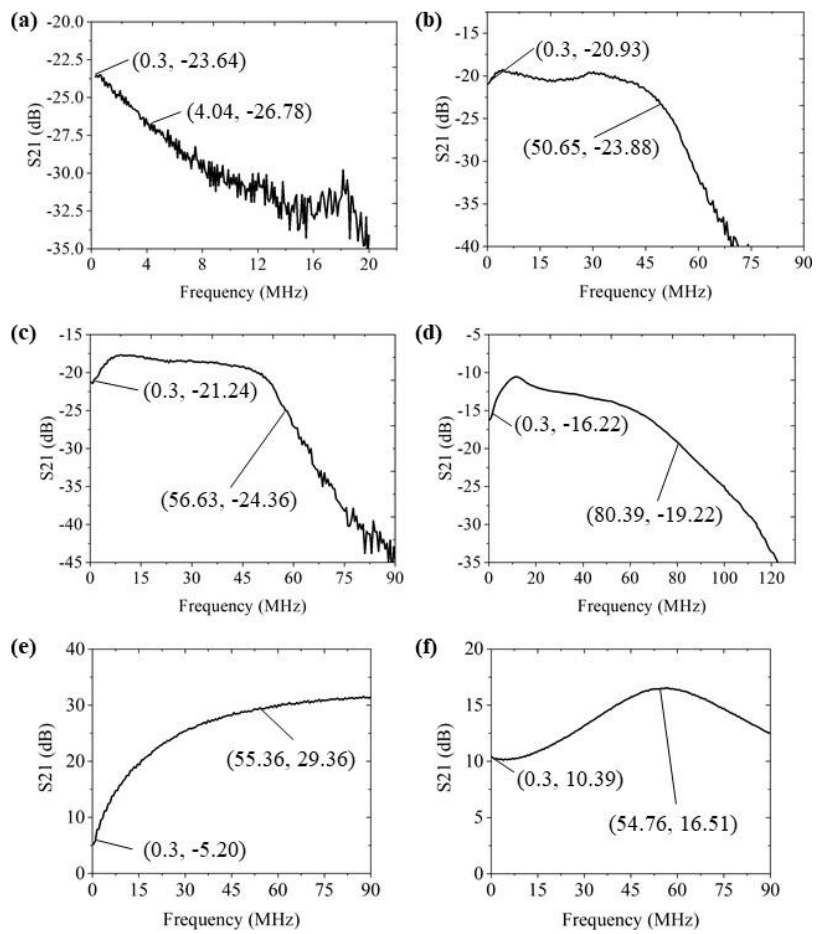

Fig. 8 Frequency response (a) only LED and photodetector (b) with post-equalization (c) with pre-emphasis (d) with both pre-emphasis and post-equalization (e) pre-emphasis circuit (f) post-equalization circuit.

At first, we measured the frequency response of each system denoted using S21. The results of the experiments are illustrated in Fig. 8. In all the experiments, a blue-filter is used. From Fig. 8(a), we can note that the $3-\mathrm{dB}$ bandwidth of LED is merely $4 \mathrm{MHz}$. As depicted in Fig. 8(b) and (c), the post-equalization and pre-emphasis circuits enhance the $3-\mathrm{dB}$ bandwidth to $50 \mathrm{MHz}$ and $56 \mathrm{MHz}$, respectively. This validates our theoretical analysis provided in Sect. 4 that the bandwidth of the pre-emphasis system can be larger than that of the post-equalization system due to the transmission loss and noise of the front-stage circuit in the 


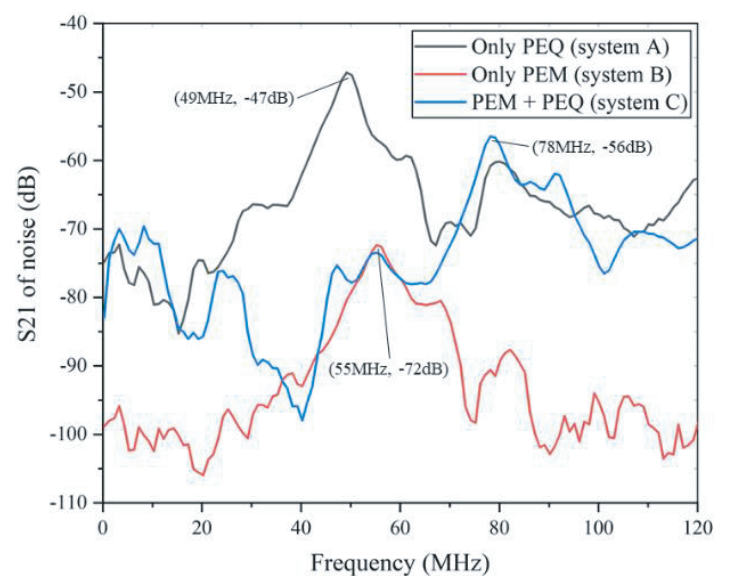

Fig. 9 Noise performance of three VLC systems.

latter system. Figure 8(d) shows the result of the system with both pre-emphasis and post-equalization circuits. We can observe from the figure that the bandwidth of this system can reach up to $80 \mathrm{MHz}$. This implies that exploiting the two circuits together can result in a remarkable performance improvement. In order to verify the analysis results, the value of $\left|H_{P E M}(\omega)\right|^{2}$ and $\left|H_{P E Q}(\omega)\right|^{2}$ is shown in Fig. 8(e) and Fig. 8(f). Since the output power of network analyzer is $0 \mathrm{dBm}$, the value of S21 in Fig. 8(a) equals to the value of $\left|H_{P}(\omega)\right|^{2}$, the value of S21 in Fig. 8(e) equals to the value of $\left|H_{P E M}(\omega)\right|^{2}$, the value of S21 in Fig. 8(f) equals to the value of $\left|H_{P E Q}(\omega)\right|^{2}$.

Next, the noise performance of three different VLC systems is measured. We still used the network analyzer in the noise measurement. However, the visible light signal is blocked in this experiment, only the noise is measured. The experimental result is shown in Fig. 9. Since the output power of network analyzer is $0 \mathrm{dBm}$, the value of $\mathrm{S} 21$ equals to the value of noise power. The experimental result is the sum of white noise, shot noise and thermal noise.

Based on the Eq. (11), Eq. (16), Eq. (21), and the experimental results, the value of $N_{P}, N_{P E M}$ and $N_{P E Q}$ can be estimated. The estimated order of magnitude of $N_{P}$ is from $10^{-1} \mathrm{~mW}$ to $10^{-3} \mathrm{~mW}$. The estimated order of magnitude of $N_{P E M}$ and $N_{P E Q}$ is from $10^{-5} \mathrm{~mW}$ to $10^{-8} \mathrm{~mW}$. Therefore, $N_{P}$ is much larger than $N_{P E M}$ and $N_{P E Q}$, which means the signal path performance is bad.

As shown in Fig. 9, the power of noise is very weak. For example, $-60 \mathrm{dBm}$ equals to $10^{-6} \mathrm{~mW}$. On this condition, the experimental result is very sensitive to ambient electromagnetic interference. Even the curvature of the cable would affect the experimental result. Take the fluctuation of the curve into consideration, we should focus on the trend of the curve.

The power of noise reaches the maximum value around the resonant frequency of each system. For the low frequency region $(0-30 \mathrm{MHz})$, the noise performance of system A and system C is similar. The noise of the only active pre-emphasis VLC system is the lowest. The noise of the VLC system with only post-equalization is the highest. We can draw the conclusion that $N_{B}<N_{C}<N_{A}$ (the preemphasis in this experiment is active). The experimental result is the same as our theoretical analysis, which is summarized above in Table 2 .

\section{Conclusion}

In this paper, we discussed the relationship between the noise and bandwidth of VLC transceivers with pre-emphasis and post-equalization circuits. We constructed a theoretical model and derived expressions for the bandwidth and $\mathrm{S} / \mathrm{N}$ of three different VLC systems. Furthermore, using theoretical analysis and experimental results, we proved that the bandwidth of the system employing both pre-emphasis and equalization is $\sqrt{2}$ times that of the system using a single pre-emphasis or post-equalization circuit. Based on the theoretical analysis and experimental verification, we can draw the conclusion that the VLC system with only active preemphasis shows the lowest noise, which is a good choice for low noise system. In the VLC system with pre-emphasis, signal strength is improved at the beginning, thereby avoiding the impact of noise in subsequent stages. The analysis provided in this paper facilitates a better design method for VLC systems.

\section{Acknowledgments}

This work was supported by the National Key Research and Development Program of China (2017YFB0403602).

\section{References}

[1] A. Jovicic, J. Li, and T. Richardson, "Visible light communication: Opportunities, challenges and the path to market," IEEE Commun. Mag., vol.51, no.12, pp.26-32, Dec. 2013. DOI: 10.1109/MCOM.2013.6685754

[2] L.U. Khan, "Visible light communication: Applications, architecture, standardization and research challenges," Digital Communications and Networks, vol.3, no.2, pp.78-88, May 2017. DOI: 10.1016/j.dcan.2016.07.004

[3] M. Ayyash, H. Elgala, A. Khreishah, V. Jungnickel, T. Little, S. Shao, M. Rahaim, D. Schulz, J. Hilt, and R. Freund, "Coexistence of WiFi and LiFi toward 5G: Concepts, opportunities, and challenges," IEEE Commun. Mag., vol.54, no.2, pp.64-71, Feb. 2016. DOI: 10.1109/MCOM.2016.7402263

[4] D. Tsonev, S. Videv, and H. Haas, "Light fidelity (Li-Fi): Towards all-optical networking," Proc. SPIE, vol.9007, no.5, p.900702, Feb. 2014. DOI: $10.1117 / 12.2044649$

[5] H. Haas, L. Yin, Y. Wang, and C. Chen, "What is LiFi?" J. Lightwave Technol., vol.34, no.6, p.1533, 2016. DOI: 10.1109/JLT.2015.2510021

[6] Y. Wang and H. Haas, "Dynamic load balancing with handover in hybrid Li-Fi and Wi-Fi networks," J. Lightwave Technol., vol.33, no.22, pp.4671-4682, Nov. 2015. DOI: 10.1109/JLT.2015.2480969

[7] H.L. Minh, D. O’Brien, G. Faulkner, L. Zeng, K. Lee, D. Jung, and Y. Oh, "High-speed visible light communications using multipleresonant equalization," IEEE Photon. Technol. Lett., vol.20, no.14, pp.1243-1245, July 2008. DOI: 10.1109/LPT.2008.926030

[8] J. Grubor, S.C. J. Lee, K.-D. Langer, T. Koonen, and J.W. Walewski, "Wireless high-speed data transmission with phosphorescent whitelight LEDs," 33rd ECEOC, 2007. 
[9] T. Kishi, H. Tanaka, Y. Umeda, and O. Takyu, "A high-speed LED driver that sweeps out the remaining carriers for visible light communications," J. Lightwave Technol., vol.32, no.2, pp.239-249, Jan. 2014. DOI: 10.1109/JLT.2013.2292896

[10] M.T. Alresheedi, A.T. Hussein, and J.M.H. Elmirghani, "Uplink design in VLC systems with IR sources and beam steering," IET Commun., vol.11, no.3, pp.311-317, 2017. DOI: 10.1049/ietcom.2016.0495

[11] D. Tsonev, H. Chun, S. Rajbhandari, J.J.D. McKendry, S. Videv, E. Gu, M. Haji, S. Watson, A.E. Kelly, G. Faulkner, M.D. Dawson, H. Haas, and D. O'Brien, "A 3-Gb/s single-LED OFDMbased wireless VLC link using a gallium nitride $\mu$ LED," IEEE Photon. Technol. Lett., vol.26, no.7, pp.637-640, April 2014. DOI: 10.1109/LPT.2013.2297621

[12] R.X.G. Ferreira, E. Xie, J.J.D. McKendry, S. Rajbhandari, H. Chun, G. Faulkner, S. Watson, A.E. Kelly, E. Gu, R.V. Penty, I.H. White, D.C. O'Brien, and M.D. Dawson, "High bandwidth GaN-based micro-LEDs for multi-Gb/s visible light communications," IEEE Photon. Technol. Lett., vol.28, no.19, pp.2023-2026, Oct. 2016. DOI: 10.1109/LPT.2016.2581318

[13] S. Rajbhandari, J.J.D. McKendry, J. Herrnsdorf, H. Chun, G. Faulkner, H. Haas, I.M. Watson, D. O’Brien, and M.D. Dawson, "A review of gallium nitride LEDs for multi-gigabit-per-second visible light data communications," Semicond. Sci. Technol., vol.32, no.2, p.023001, Jan. 2017. DOI: 10.1088/1361-6641/32/2/023001

[14] H.L. Minh, D. O’Brien, G. Faulkner, L. Zeng, K. Lee, D. Jung, and Y. Oh, "80 Mbit/s visible light communications using pre-equalized white LED," 34th ECOC, p.6.09, Brussels, Belgium, Sept. 2008. DOI: 10.1109/ECOC.2008.4729532

[15] N. Fujimoto and H. Mochizuki, "477 Mbit/s visible light transmission based on OOK-NRZ modulation using a single commercially available visible LED and a practical LED driver with a pre-emphasis circuit," OFC/NFOEC, Anaheim, California United States, p.JTh2A.73, March 2013. DOI: 10.1364/NFOEC. 2013.JTh2A.73

[16] C.-H. Yeh, Y.-L. Liu, and C.-W. Chow, "Real-time white-light phosphor-LED visible light communication (VLC) with compact size," Opt. Express, vol.21, no.22, pp.26192-26197, Nov. 2013. DOI: 10.1364/OE.21.026192

[17] H. Li, X. Chen, J. Guo, D. Tang, B. Huang, and H. Chen, " $200 \mathrm{Mb} / \mathrm{s}$ visible optical wireless transmission based on NRZ-OOK modulation of phosphorescent white LED and a pre-emphasis circuit," Chin. Opt. Lett., vol.12, no.10, p.100604, Oct. 2014. DOI: 10.3788/COL201412.100604

[18] H. Li, X. Chen, J. Guo, Z. Gao, and H. Chen, "An analog modulator for $460 \mathrm{Mb} / \mathrm{s}$ visible light data transmission based on OOKNRS modulation,” IEEE Wireless Commun., vol.22, no.2, pp.68-73, April 2015. DOI: 10.1109/MWC.2015.7096287

[19] X. Huang, J. Shi, J. Li, Y. Wang, and N. Chi, "A Gb/s VLC transmission using hardware pre-equalization circuit," IEEE Photon. Technol. Lett., vol.27, no.18, pp.1915-1918, June 2015. DOI: 10.1109/LPT.2015.2445781

[20] X. Li, N. Bamiedakis, X. Guo, J.J.D. McKendry, E. Xie, R. Ferreira, E. Gu, M.D. Dawson, R.V. Penty, and I.H. White, "Wireless visible light communications employing feed-forward pre-equalization and PAM-4 modulation,” J. Lightwave Technol., vol.34, no.8, pp.2049_ 2055, April 2016. DOI: 10.1109/JLT.2016.2520503

[21] H. Zhang, A. Yang, L. Feng, and P. Guo, "Gb/s real-time visible light communication system based on white LEDs using T-bridge cascaded pre-equalization circuit," IEEE Photon. J., vol.10, no.2, p.7901807, March 2018. DOI: 10.1109/JPHOT.2018.2812759

[22] H.L. Minh, D. O’Brien, G. Faulkner, L. Zeng, K. Lee, D. Jung, Y. Oh, and E.T. Won, "100-Mb/s NRZ visible light communications using a post-equalized white LED," IEEE Photon. Technol. Lett., vol.21, no.15, pp.1063-1065, Aug. 2009. DOI: 10.1109/LPT.2009.2022413

[23] H. Li, X. Chen, B. Huang, D. Tang, and H. Chen, "High bandwidth visible light communications based on a post-equalization circuit,' IEEE Photon. Technol. Lett., vol.26, no.2, pp.119-122, Jan. 2014 DOI: 10.1109/LPT.2013.2290026

[24] H. Li, X. Chen, J. Guo, and H. Chen, "A 550 Mbit/s real-time visible light communication system based on phosphorescent white light LED for practical high-speed low-complexity application," Opt. Express, vol.22, no.22, pp.27203-27213, Nov. 2014. DOI: 10.1364/OE.22.027203

[25] H. Li, Y. Zhang, X. Chen, C. Wu, J. Guo, Z. Gao, W. Pei, and H. Chen, "682 Mbit/s phosphorescent white LED visible light communications utilizing analog equalized 16QAM-OFDM modulation without blue filter," Opt. Commun., vol.354, pp.107-111, 2015. DOI: 10.1016/j.optcom.2015.05.033

[26] Y. Wang, Y. Wang, N. Chi, J. Yu, and H. Shang, "Demonstration of $575-\mathrm{Mb} / \mathrm{s}$ downlink and 225-Mb/s uplink bidirectional SCM-WDM visible light communication using RGB LED and phosphor-based LED,” Opt. Express, vol.21, no.1, pp.1203-1208, Jan. 2013. DOI: 10.1364/OE.21.001203

[27] N. Fujimoto and S. Yamamoto, "The fastest visible light transmissions of $662 \mathrm{Mb} / \mathrm{s}$ by a blue LED, $600 \mathrm{Mb} / \mathrm{s}$ by a red LED, and $520 \mathrm{Mb} / \mathrm{s}$ by a green LED based on simple OOK-NRZ modulation of a commercially available RGB-type white LED using preemphasis and post-equalizing techniques," ECOC, Cannes, France, Sept. 2014. DOI: 10.1109/ECOC.2014.6963895

[28] Y. Wang, L. Tao, X. Huang, J. Shi, and N. Chi, "8-Gb/s RGBY LED-based WDM VLC system employing high-order CAP modulation and hybrid post equalizer,' IEEE Photon. J., vol.7, no.6, pp.1-7, Dec. 2015. DOI: 10.1109/JPHOT.2015.2489927

[29] Y. Wang, L. Tao, X. Huang, J. Shi, and N. Chi, "Enhanced performance of a high-speed WDM CAP64 VLC system employing volterra series-based nonlinear equalizer," IEEE Photon. J., vol.7, no.3, p.7901907, June 2015. DOI: 10.1109/JPHOT.2015.2436911

[30] X. Huang, S. Chen, Z. Wang, J. Shi, Y. Wang, J. Xiao, and N. Chi, "2.0-Gb/s visible light link based on adaptive bit allocation OFDM of a single phosphorescent white LED," IEEE Photon. J., vol.7, no.5, p.7904008, Oct. 2015. DOI: 10.1109/JPHOT.2015.2480541

[31] A.M. Khalid, G. Cossu, R. Corsini, P. Choudhury, and E. Ciaramella, "1-Gb/s transmission over a phosphorescent white LED by using rate-adaptive discrete multitone modulation,’ IEEE Photon. J., vol.4, no.5, p.1465, Oct. 2012. DOI: 10.1109/JPHOT.2012.2210397

[32] S. Haykin, ed., Communication Systems, John Wiley, 2000.

[33] H.T. Friis, "Noise figures of radio receivers," Proc. IRE, vol.32, no.7, pp.419-422, July 1944. DOI: 10.1109/JRPROC.1944.232049

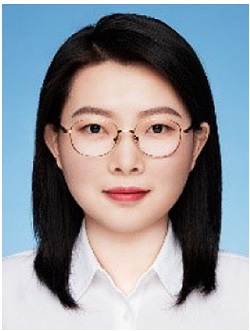

Dong Yan received the B.S. and M.S degrees in School of Electrical and Information Engineering, Tianjin University in 2011 and 2014, respectively. She is currently working toward the Ph.D. degree at Tianjin University. Her research interests include visible light communication, CMOS optoelectronic integrated circuit, and optical wireless communication transceiver. 


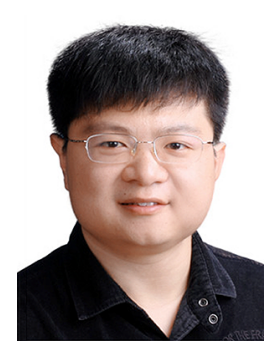

Xurui Mao received the B.S. and M.S. degrees from Tianjin University, Tianjin, China, and the Ph.D. degree from University of Chinese Academy of Sciences, Beijing, China. He is currently an associate professor with the Institute of Semiconductors, Chinese Academy of Sciences. His current research interests include optoelectronic integrated circuits and novel electronic devices.

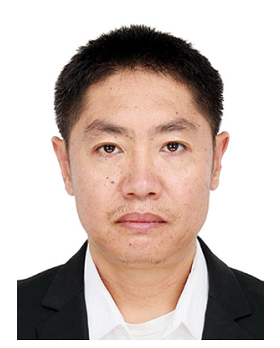

Sheng Xie is an associate professor of the school of microelectronics, Tianjin University, China. He received the Ph.D. degree from Xiamen University in 2006. His research interests include photo electronic device, CMOS optoelectronic integrated circuit receiver, wireless communication transceiver, and UHF radio frequency identification technology and applications.

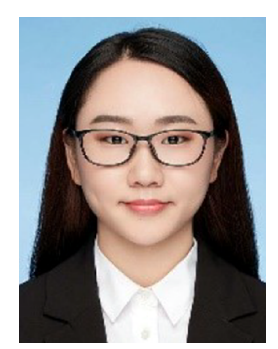

Jia Cong received the B.S. degrees Physics from Shandong Normal University in 2012 and M.S. degrees in Optical Engineering from Nankai University in 2014. She now is a doctoral student majoring in Information and Communication Engineering at Tianjin University. Her research interests include OEIC Receiver Chip for Visible Light Communication

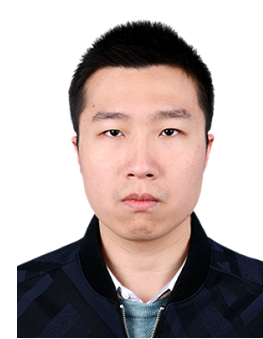

Dongqun Han received the B.S. degree in microelectronics at Anhui University in 2015, the M.S. degree in Electrical and Information Engineering at Tianjin University in 2018. His research interests include visible light communication receiver design and post-equalization technique.

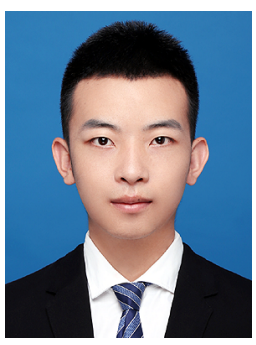

Yicheng Wu received the B.S. and M.S. degrees in School of Electrical and Information Engineering, Tianjin University in 2015 and 2018, respectively. His research interests include high power LED driver design and integrated visible light communication transmitter. 